\title{
Cranial morphometric and fine scale genetic variability of two adjacent Mastomys natalensis (Rodentia: Muridae) populations
}

\author{
Aude LALIS, Michel BAYLAC, Jean François COSSON, Rhodes H. MAKUNDI, \\ Robert S. MACHANG'U and Christiane DENYS
}

Lalis A., Baylac M., Cosson J. F., Makundi R. H., Machang'u R. S. and Denys C. 2009. Cranial morphometric and fine scale genetic variability of two adjacent Mastomys natalensis (Rodentia: Muridae) populations. Acta Theriologica 54: 000-000.

The objective of this multidisciplinary project was to study the intra-specific morphometric and genetic variability between two adjacent populations of Mastomys natalensis Smith, 1834 living in different environments. The study of micro-evolutionary processes at work by using geometrical morphometrics allowed us to define two groups, characterized by different features of the skull shape. Using molecular microsatellites analysis, we showed that the two populations exchanged high gene flow and could be considered as a single panmictic unit. These results suggest that this widely-distributed species exhibits a local population-level differentiation in shape variation of skulls, probably due to different ecological situations. We speculate that the variability in the cranial characteristics (connected with the feeding ability) could reveal a local adaptation preferentially based on the food availability. We propose an explanation linking the shape differences to the fitness gain in the exploitation of resources available in the two environments. Since we suggest a potential differentiation process between populations, we believe that the two groups constitute even better models to understand the factors involved in the early stages of local adaptations. The aim of such studies is to provide a better understanding of the economical importance of this species and its remarkable capability to proliferate.

UMR CNRS 5202 - USM 601, Origine, Structure et Evolution de la Biodiversité, Département Systématique et Evolution, Muséum National d'Histoire Naturelle, CP 51, 55 rue Buffon, 75005 Paris, France, e-mail: lalis@mnhn.fr (AL, MB, CD); Plate-forme Morphométrie MNHN-CNRS IFR 10145, rue Buffon F-75005 Paris, France (AL, MB); Centre de Biologie et Gestion des Populations (UMR22), INRA, Campus International de Baillarguet, CS30016, 34988 Montferrier sur Lez cedex, France (JFC); Rodent Research Project, Sokoine University of Agriculture, Morogoro, Tanzania (RHM, RSM)

Key words: Mastomys natalensis, intra-specific variability, skull shape variation, geometric morphometrics, genetic differentiation, microsatellites

\section{Introduction}

The African endemic genus Mastomys, Thomas (1915) or multimammate rat (Rodentia,
Muridae) is responsible for important agriculture and public health problems by causing significant damages to cultivated fields and by transmitting numerous serious diseases $(\mathrm{eg}$, 
the bacteria Yersina pestis, the etiologic agent of plague and Lassa fever virus) (Monath et al. 1974, Kilonzo et al. 1992, Gratz 1997, Mwanjabe and Leirs 1997, Demby et al. 2001, Mwanjabe et al. 2002). Mastomys natalensis Smith, 1834 is a common anthropophilous species and has the largest geographic distribution within the genus (Granjon et al. 1997). It shows all the characteristics of a pest species: high breeding rate, widespread outbreaks, strong capacity of dispersion and colonization (Leirs 1995). In Tanzania, some populations of $M$. natalensis undergo irregular population explosions with densities as high as 1400 rats per hectare (Mwanjabe et al. 2002). Pest control on fields becomes a necessity because the majority of farmers are smallholders (Mwanjabe and Leirs 1997). The ecological opportunism of $M$. natalensis and its tendency to be dominant in the rodent communities define this species as a good model in the study and understanding of the distribution mechanisms by population genetics. Only one population genetic analysis on $M$. natalensis on a small geographic scale using microsatellites has been realized in the past ( $P$. van Hooft and J. F. Cosson, pers. comm.). Although of great interest in the frame of pest control and human disease studies, gene flow rates within and between populations of $M$. natalensis are not well-known.

Variation in phenotypic and genetic traits across geographic space is an ubiquitous phenomenon exhibited by most organisms (Monteiro et al. 2003) and the relationship between genotype and phenotype is a recurrent question in modern biology. The descriptions of morphological versus genetic characters in the patterns of variations within and between populations are fundamental for defining boundaries of independent evolutionary units in nature (Dos Reis et al. 2002a). The study of phenotypic differentiation is relevant, not only as an approximation to the underlying genetic differentiation, but also because realized phenotypes result from the interfacing between morphogenetic rules, ecological conditions and deterministic and stochastic evolutionary forces (Monteiro et al. 2003). For the phenotype, the material basis of the differences is defined in terms of size and shape (Monteiro et al. 2003).
With different techniques of geometrical morphometrics which allow a quantification of shape variations, some approaches have been possible in the discrimination and classification of species (Dobigny et al. 2002, Cordeiro et al., in press and in analysis of shape evolution (Auffray et al. 1996, Mullin et al. 2004, Taylor et al. 2005). The geometrical morphometric approach (Bookstein 1991, 1996, Rohlf 2000) has already been successfully applied to investigate aspects of morphological variation in the echimyid rodent, Trichomys apereoides (Monteiro et al. 1999, Duarte et al. 2000, Dos Reis et al. 2002a, b), in Mus musculus (Auffray et al. 1996, Alibert et al. 1997, Debat et al. 2000), in Dasymys (Mullin et al. 2004) and in Otomys saundersiae (Taylor et al. 2005).

The objectives of this study are to elucidate qualitatively and quantitatively intra-specific morphological and genetic variability at a populational scale. Patterns of local variation in cranial shape among populations of the rodent species $M$. natalensis in relation to genetic differentiation are investigated using the framework of geometrical morphometrics and microsatellites analysis.

\section{Material and methods}

\section{Samples}

Specimens came from the rodents collection of Museum National d'Histoire Naturelle de Paris. All individuals were collected in July 2000 at the edge of the Selous Game Reserve (Tanzania). Cytogenetic analyses were conducted in the field and in laboratory for accurate identification of species M. natalensis (Denys et al., in press). Two different habitats have been sampled with the same trapping effort (100 Sherman traps per locality for three consecutive nights). The sampling effort yielded 137 Mastomys individuals (71 males, 66 females). These individuals came from two distinct populations separated by $5 \mathrm{~km}$. The first population (107 individuals) lived in an agricultural area (dry maize field) close to a village, a typical habitat for the species in Tanzania. The second population (30 individuals) was located in a swampy area with a permanent waterhole in a "degraded miombo" (savanna of fruit trees), a less typical habitat for the species. 


\section{Age determination}

There are numerous methods for age estimation, both relative and absolute (reviewed by Morris 1972, Pucek and Lowe 1975, Poulet 1980). Among them, the majority of age criteria based on external (weight, body length) or internal morphological (bone development, tooth wear, eye lens weight) characters have been proven to be imprecise (Le Louarn 1971, Lalis et al. 2006). Although body weight is not always directly and linearly related to age in Mastomys, it can be used as an effective age estimator (Leirs 1995). It was thus possible to group the animals in three "reproductive age groups" according to their weight (Leirs 1995): “Juveniles" (animals below 25 grams; they represent the very young individuals), "Sub adults" (sexually mature, weighting between 25 and 40 grams) and "Adults" (above 40 grams).

\section{Geometric morphometric analysis}

\section{Skull landmarks}

Our sampling included 129 individuals for the dorsal side of the cranium and 104 individuals for the ventral side, and excluded all specimens with missing landmarks. Three-dimensional landmarks, assumed to be homologous from individual to individual, were identified for the dorsal and ventral sides of the skull. They included points of suture between bones or points of intersection between sutures (25 landmarks for dorsal side and 35 landmarks for ventral side; Fig. 1). Each individual skull was digitalized for each side with the Reflex Microscope (reflex Measurements Ltd.).

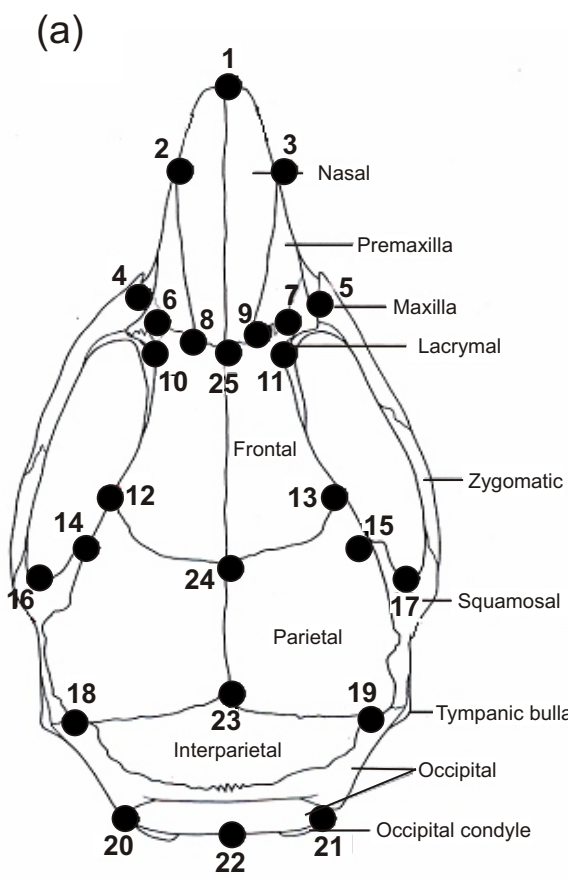

(b)

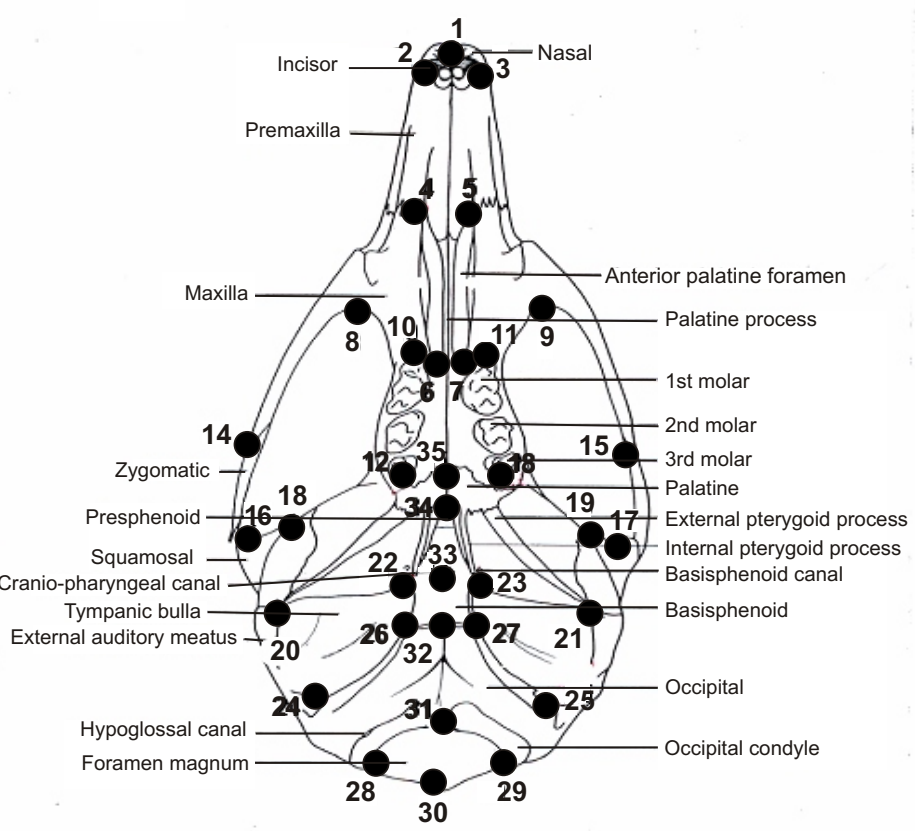

Fig 1. Landmark location for a) the dorsal and b) the ventral views of the skull of Mastomys natalensis (Cook 1965). Dorsal view: tip of the nasals (1), most anterior points at nasal - premaxillary suture $(2,3)$, anterior projection of zygomatic $(4,5)$, intersection of premaxilla and frontal $(6,7)$, nasal - frontal suture $(8,9,25)$, suture of premaxilla and maxilla over lachrymal capsule $(10,11)$, frontal - parietal suture $(12,13,24)$, most medial point at interorbital constriction (14, 15), back of zygomatic notch $(16,17)$, intersection of parietal - interparietal and supraoccipital sutures 18, 19), back of the lateroccipital protuberances $(20,21,22)$, midpoint of parietal interparietal suture (23). Ventral view: tip of the nasals (1), antero-lateral extremity of left or right incisive alveolus $(2,3)$, anterior palatine foramen $(4,5)$, posterior palatine foramen $(6,7)$, back of zygomatic plate $(8,9)$, intersection between left or right anterior end of molar and mandible $(10,11)$, intersection between left or right posterior end of molar and mandible $(12,13)$, suture between jugal and squamosal in the left or right zygomatic arch (14, 15), front of glenoid fossa on squamosal root of zygomatic arch $(16,17)$, suture between left or right parietal and squamosal (18, $19)$, back of external opening of auditory bullae $(20,21)$, lateral points of sphenoccipital suture $(22,23)$, styloinastoid foramina at the posterior border of external auditory meatus $(24,25)$, anterior extremity of occipital $(26,27)$, posterior intersection between foramen magnum and occipital condyle $(28,29)$, posterior extremity of foramen magnum (30), anterior extremity of foramen magnum (31), anterior extremity of occipital (32), cranio - pharyngeal canal (33), contact point between palatine and presphenoid (34), contact point between maxilla and palatine (35). 


\section{Morphometric analysis}

Ventral and dorsal landmarks configurations were superimposed separately by a least-squares generalized Procrustes algorithm. The generalized Procrustes superimposition procedure scales, translates and rotates the configurations to minimize the summed squared distances between corresponding landmarks (Rohlf and Slice, 1990). The size measurement was calculated by the log of centroid size, defined as the square root of the summed squared distances from each landmark to the overall centroid. Landmark configurations were used to compute consensus configuration for dorsal and ventral sides of the skull for population samples of Mastomys natalensis as the grand mean of aligned specimens from all populations. Residuals from the mean landmark locations (= Procrustes residuals) together with centroid size constitute the shape used in the statistical analyses.

\section{Multivariate statistical analyses}

The size patterns of variability were analysed by analysis of variance (ANOVA) related to age, sex and locality (and the interactions). Regression parameters allow prediction and visualization of the shape changes in relation to the specified variable. We used this approach to visualize the shape variation along the canonical axes, as well as for the allometrical patterns modeling (Flury 1984, Airoldi and Flury 1988) in order to describe the allometry-free shape differences among populations. These allometry-free patterns were submitted to a discriminant analysis using multivariate analysis of covariance (MANCOVA) with $\log 10$ centroid size as a covariate. The dorsal and ventral views have been combined on a single dataset for the statistical analyses (Canonical Variate Analyses) by using the $\mathrm{R}$ language (R.2.0.1, 2004). Superimpositions and visualizations were calculated with the 'Rmorph' library for R (Baylac 2007). Due to the small samples sizes, shape analyses were realized using the 10 first principal components (PCs) extracted from Procrustes residuals which explained $73.8 \%$ of the total variance for the ventral side and $79.4 \%$ for the dorsal side. This number of PC was selected to minimize the cross-validated misclassification percentages (Baylac and Friess 2005).

\section{Genetic markers analysis}

\section{Sample collection}

We used 31 samples of $M$. natalensis which came from the two studied populations (23 for population 1 and 8 for population 2). Total DNA was extracted from liver and spleen preserved in $70 \%$ ethanol using the Dnease Tissue Kit (Qiagen).

\section{Microsatellite analysis}

For genotyping, we used a set of 10 microsatellite markers with dinucleotide repeats and specially developed for Mastomys species (Galan et al. 2004). The microsatellites were amplified in a single multiplex PCR reaction with the Qiagen Multiplex PCR kit following manufacturer's instruc- tions. The PCR products were tested on monocapillary ABI PRISM 310 Genetic Analyser and GENESCAN analysis software (Applied Biosystems).

\section{Statistical analysis of microsatellite data}

Mean number of alleles per locus, observed $\left(H_{o}\right)$ and expected $\left(H_{e}\right)$ heterozygosity (Nei 1987) were calculated over all loci at each sampling location using the software GENETIX v4.04 (Belkhir 2001). Tests for departures from Hardy-Weinberg equilibrium, for genotypic linkage disequilibrium and independence between populations, and allele frequency were performed for all populations using the probability tests in GENEPOP v3.3 software (Raymond and Rousset 1995). Markov chain method was used to provide an unbiased estimation of the exact $p$-value (more than 5 alleles) and to test genotypical divergence among populations for all loci and population pairs. $F$-statistics: $F_{S T}$ (population differentiation) and $F_{I S}$ (heterozygote deficit, Watterson 1978) were calculated according to Weir and Cockerham (1984) using GENEPOP software, and the significance of Fst estimates was determined using a permutation test implemented in the GENETIX v4.04 package (1000 permutations, one-sided test of the null hypothesis of no genetic difference among the groups of populations).

\section{Results}

\section{Age determination: "Body weight" method}

The individuals were grouped into three age classes depending on their body weight. The age class distribution of the two populations showed that the majority of individuals for the two samplings belongs to the juvenile class $(93 / 137$ individuals). A Fisher exact test of significance showed that the two populations were not significatively different in terms of age ( $p=$ $0.1079)$ and $\operatorname{sex}(p=0.1138)$.

\section{Geometrical morphometrics}

Size variation

Neither 'population' (ANOVA: $F=0.2188, p=$ $0.7896)$ nor 'sex' $(F=2.3874, p=0.0657)$ parameters were significant on size differences (Table $1)$. As expected, centroid size was strongly correlated to age $(F=78.82, p<0.001)$.

\section{Shape variation}

The results of multivariate analysis of covariance (Table 1) on geometrical shape with 
Table 1. ANOVA on centroid size and MANCOVA on Procrustes residuals from two populations of Mastomys natalensis. Ventral and dorsal views were pooled for analyses. Log transformed centroid size was used as covariate to account for allometric effects of size variation. Significance codes: $* * * * 0, * * *-0.001, * *-0.01, *-0.05$.

\begin{tabular}{|c|c|c|c|c|c|c|c|}
\hline \multicolumn{4}{|c|}{ ANOVA - centroid size 2 Views (dorsal + ventral) } & \multicolumn{4}{|c|}{ MANCOVA - skull shape } \\
\hline Variables & $\mathrm{df}$ & $F$-values & $P$-values & Covariates & $\mathrm{df}$ & $F$-values & $P$-values \\
\hline Pop & 1 & 0.2188 & 0.7896 & Pop & 1 & 3.1285 & $0.0009 * * *$ \\
\hline Sex & 1 & 2.3874 & 0.0657 & Sex & 1 & 1.0155 & 1.0025 \\
\hline Age & 2 & 78.8224 & $1.588 \mathrm{e}-15^{* * *}$ & Age & 2 & 6.4956 & $1.478 \mathrm{e}-11^{* * * *}$ \\
\hline Interaction Pop.Sex & 1 & 0.1458 & 0.8763 & Log centroid size & 1 & 0.4159 & 0.9563 \\
\hline Interaction Sex.Age & 2 & 1.4687 & 1.5334 & Interaction Pop.Sex & 1 & 0.7466 & 0.6148 \\
\hline \multirow[t]{2}{*}{ Interaction Pop.Age } & 2 & 0.0878 & 0.8756 & Interaction Sex.Age & 2 & 1.7014 & 0.5499 \\
\hline & & & & Interaction Pop.Age & 2 & 1.2520 & 0.1856 \\
\hline
\end{tabular}

"population", "sex", "age" and "centroid size" as independent variables, and their interaction revealed two distinct patterns in shape variation. The "population" and "age" factors were highly significant (MANCOVA: $F=3.1285, p<0.001 ; F$ $=6.4956, p<0.001$ respectively). The results for the "population" parameter presented low misclassification percentages (dorsal: $25.20 \%$, ventral: $18.75 \%$ ) (Table 2). The low overall misclassification rates were mainly due to the larger sample size in population 1 . The "sex" parameter was not significant (MANCOVA: $F=$ $1.0155, p=1.0025$ ) (Table 1). For the 'population' factor, shape transformations along the discriminant axe 1 (PC1) accounting for $32 \%$

Table 2. Re-classification percentages of individuals of Mastomys natalensis to two populations (Pop1 and Pop2) according to discriminant analyses of skull shape using cross-validation.

\begin{tabular}{lccc}
\hline \multicolumn{4}{c}{ Variable : population } \\
\hline From/To & Pop1 & Pop2 & Numbers \\
\hline \multicolumn{4}{c}{ Ventral side } \\
Pop1 & 84 & 5 & 89 \\
Pop2 & 16 & 7 & 23 \\
Total of missclassified individuals (\%) $=18.75$ & \\
\multicolumn{4}{c}{} \\
Pop1 & \multicolumn{4}{c}{ Dorsal side } \\
Pop2 & 82 & \\
Total of missclassified individuals $(\%)=25.20$ \\
\end{tabular}

(ventral view) and $27 \%$ (dorsal view) of the variation in skull shape, summarized the variation in the antero-posterior orientation. PC2 accounted for $11.6 \%$ (ventral view) and $8.5 \%$ (dorsal view) of the variance which illustrated with lateral deformation at the level of the tympanic bulla. Figure 2 showed the visualizations of Procrustes superimpositions for the landmarks according the "population" (mean shape of population 1 versus mean shape of population 2). In ventral view, the population 1 seemed to have a skull relatively shorter and wider at the level of the tympanic bulla. Moreover, the posterior part of cranial shape appeared to be relatively different between both populations: the orbital limit of suture between parietal and squamosal and the extremities of tympanic bullas seemed to be organized differently in term of space disposition (Fig. 2). We also observed that the lengths of molar rows were different between the both populations since it is larger in population 1 than in population 2 (Fig. 2). In dorsal view, population 2 seemed to present a longer and thinner shape than in the population 1 with a more pronounced rostrum (contact points between nasal, premaxillary and frontal which were situated in the back of the skull) (Fig. 2). In conclusion and according to the results obtained on the 2 sides, a significant difference in the skull shape between populations was observed: a shorter cranial shape for population 1 and an important difference in the lengths of molar rows between the populations (Fig. 2). 

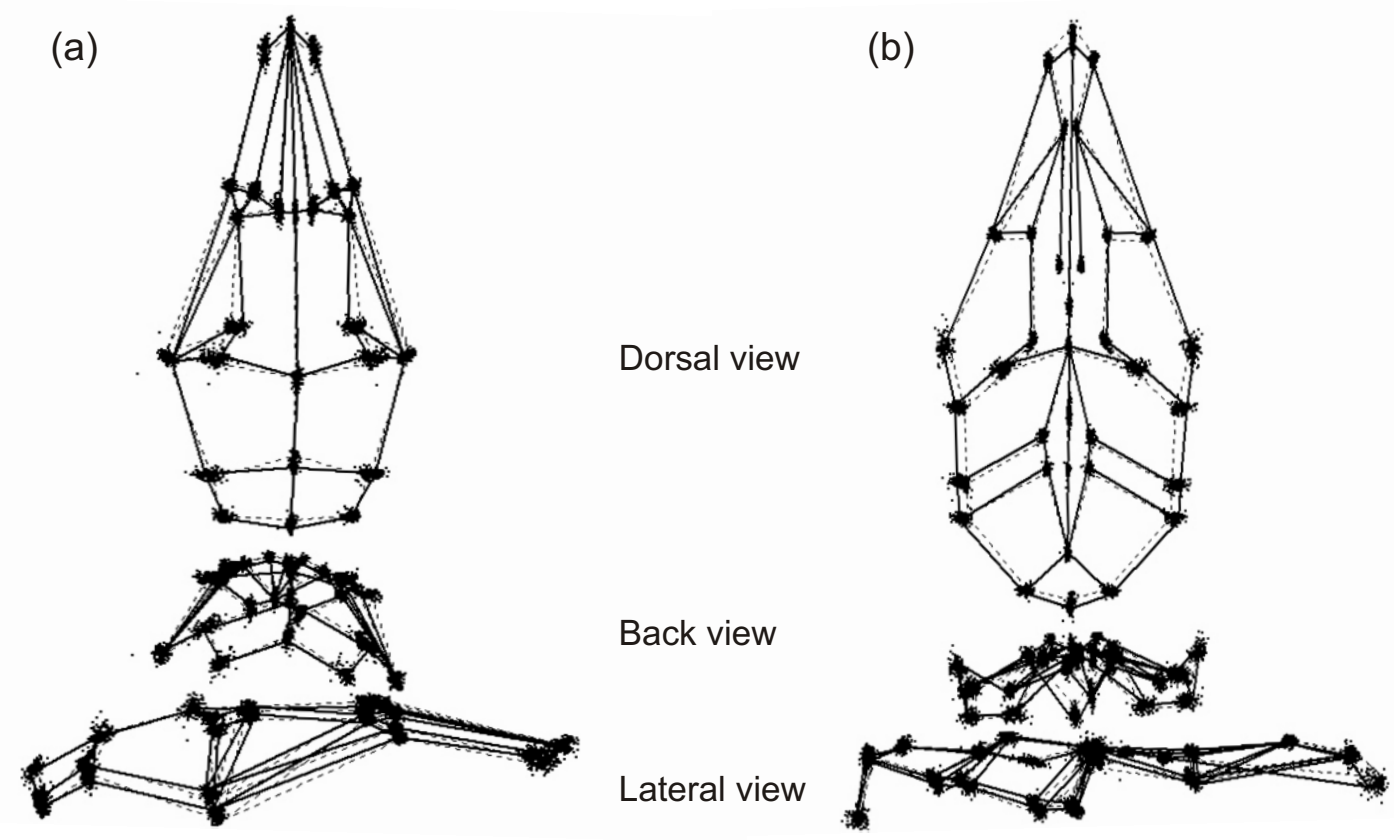

Fig. 2. Shape transformations of the skull along the discriminant axes for the two populations of Mastomys natalensis. Solid lines depict group 1; dotted lines depict group 2. Dorsal (a) and ventral (b) sides.

\section{Microsatellite study}

Number of alleles and allelic diversity

All microsatellite loci showed a high genetic diversity: the number of alleles per locus ranged from 9 to 24 with a mean number of alleles per locus $=15.3$. The mean number of alleles per locus and per population was of 10.35 (Table 3). No linkage disequilibrium between loci occurred for all mixed populations except between the two loci which displayed a low disequilibrium $(0.0441<0.05)$. However, independence among loci was assumed in the subsequent analyses.

Intrapopulation genetic diversity

The number of alleles per locus ranged from 7 to 16 for population 1 and from 4 to 12 for population 2 with a mean number of alleles per locus respectively of 11.8 and 8.9 (Table 3). The expected heterozygosity was relatively high. According to locus and population (mean number = 0.889 ), it varied between 0.884 and 0.894 (Table 3 ). Overall, the average values for expected heterozygosity were similar between localities.
All loci for each population were at Hardy Weinberg equilibrium (HWE).

Populational differentiation

$F$-statistics revealed a non-significant genotypical differentiation among populations. The $F_{S T}$ value was very low $(0.00150)$. The $F_{I S}$ value underlined the absence of Wahlund effect (a subdivided population contains fewer heterozygotes than predicted despite the fact that all subdivisions are in Hardy-Weinberg equilibrium). We applied the $F_{I S}$ test to mixed populations 1 and 2 (= hypothesis of a unique

Table 3. Genetic diversity at sampling sites.

\begin{tabular}{lccc}
\hline \multirow{2}{*}{ Population } & $\begin{array}{c}\text { Mean no. } \\
\text { allele/locus }\end{array}$ & \multicolumn{2}{c}{ Heterozygosity } \\
\cline { 3 - 4 } & & Expected $\left(H_{e}\right)$ & Observed $\left(H_{o}\right)$ \\
\hline 1-Village & 11.8 & 0.884 & 0.895 \\
2-Swamp & 8.9 & 0.894 & 0.900 \\
Mean & 10.35 & 0.889 & 0.898 \\
\hline
\end{tabular}


population) in order to test the heterozygote deficiency. We noticed a heterozygote deficiency relatively similar or inferior to those observed in the mean value for isolated populations $\left(F_{I S}\right.$ Pop1 $=-0.017$ and $F_{I S}$ Pop2 $\left.=-0.0025\right)$. The hypothesis of a panmictic population between the population 1 and 2 was confirmed.

\section{Discussion}

We have shown that the two populations were not significatively different in terms of composition in "age" and "sex". The data represented instantaneous samples of the natural population in a dry period and a drought zone. We knew that breeding was highly seasonal for $M$. natalensis. It usually starts after the rain season in Tanzania (in April) and lasts until the dry season (September) (Stenseth et al. 2001), with some births observed from May to October (Lima et al. 2003). Adults die after the breeding season and juveniles usually do not become sexually mature until the next dry season (Lima et al. 2003). The age composition of our samplings reflected the biological cycle of the species in Tanzania. Indeed, the sampling campaign took place at the beginning of July, while the juveniles of the year were present in large numbers. During the last period (July, August, and September) adults began to die progressively (Leirs et al. 1990), which explained their small representation in July. The campaign, made in July 2003, took place during a dry period (Lalis et al. 2006). The absence of rainfall until July was probably responsible for an important mortality in all age classes and an important delay of the development of surviving juveniles, which explained the large numbers of sexually immature juveniles. The observation of no gestating females during the sampling confirmed the exceptional context of the winter 2003, which was characterized by almost no breeding.

The genetic diversity index is coherent because $H_{e}$ (expected heterozygosity) values higher than 0.82 were not special for rodents as it had already been observed in Apodemus flavicollis (Gockel et al. 1997), Rattus fuscipes (Hinten et al. 1999), Peromyscus leucopus (Moss- man and Waser 2001) and Mus musculus (Yu and Peng 2002). Such an important genetic diversity seems to be related to large density populations. In the present study, genetic results showed that the two populations were not two isolated populations for breeding. $F_{S T}$ value appeared low and not - significant. The genetic differentiation between populations was not supported by differences in allelic frequencies or significant deviations from HWE. Therefore, no clear genetic differentiation between genetic pools was observed. With the microsatellite study, it appeared that the two populations exchanged high gene flow and could be considered as a single panmictic unit. The parameters of genetic diversity were probably reduced by the migration phenomenon. Indeed, P. van Hooft and J. F. Cosson (pers. comm.) have underlined the existence of an important migration between $M$. natalensis populations in Tanzania. They have showed that juveniles and adults were responsible for high migration rates. Leirs (1995) has demonstrated that the species, used to short daily distances (10-15 m), was sometimes able to cover a distance of $100 \mathrm{~m}$ per day. Since our samples were mainly constituted by juvenile individuals ( 1 to 3 months), we were in a context where the migration phenomenon could be important and could have led to a strong local $H_{e}$ and a low genetic differentiation between field plots, which was in agreement with the observed results.

In geometrical morphometrics, the statistical results demonstrated that the "sex" variable was not significant and confirmed the absence of a sexual dimorphism for our samples. In murids, sexual dimorphism has rarely been demonstrated on the basis of classical morphometrics distance analysis. For M. natalensis of South Africa, Smithers et al. (1979) showed that males were larger than females by body measurements but the statistical results were not significant. Delany (1975) raised an opposite tendency for a Ugandan population but obtained a statistically non significant result (females were larger than males), a point also demonstrated in populations in Senegal by Duplantier (1988). Size and shape change across ages. The "age" parameter was very significant in the both views (for Centroid 
size and Procrustes residuals) and probably corresponded to growth allometry. The statistical results confirmed that the "population" variable was significant and underlined a significant difference in the skull structure between populations. Two sides gave some distinct perspectives on the variation of cranial shape and on the organization of morphological variation between studied populations. On the visualizations, cranial shape of population 1 was observed to be shorter but higher than the cranial shape of population 2. With the 'allometry-free' model, the hypothesis of allometric differences between populations is directly tested and cannot explain the morphological differences between populations.

Such shape variation confirms the strong intra specific variability in $M$. natalensis. Some differences between populations were situated in articular zones, especially on the ventral side. These observations could be related to a difference in a diet and consequently in a morphological adaptation. Indeed, some multiple genetic and environmental factors influence the cranial shape but several lines of evidence suggest that changes in diet and food processing technology contribute to some proportion of variations in facial size and shape (Lieberman et al. 2004). Most experimental data on facial growth responses to masticatory loading come especially from primate studies (Beecher et al. 1983, Bouvier and Hylander 1996). However, some experiments on rats reveal that the individuals raised on soft food have smaller jaw adductor muscles, generate lower mandibular strains, and have significantly decreased anterior facial height, shorter mandibles, and smaller muscle attachment areas (Engström et al. 1986, Kiliaridis et al. 1986, Yamada and Kimmel 1991). In this study, the individuals of population 1 (village) feeds mainly on some maize, rice and seeds, which are harder than green vegetation and arthropods that were consumed by population 2 "swamp". This could explain the morphological differences but. Thus, strain and nutrition both influence jaw size, but only strain affects jaw shape. The changes in tympanic bulla underline a light asymmetry between populations. It would be interesting in our case to realize a detailed study of fluctuating asymmetry, which is generally considered as the only form of asymmetry that can serve as a useful indicator of organisms subjected to stress (Palmer and Strobeck 1986, Leary and Allendorf 1989, Marchand 2003) in order to know if the 'village' population is in a more stressful situation than the 'swamp' population.

In summary, with the geometrical morphometric study, two groups of populations living in different environments have been shown to be significantly different, based on some distinct morphological features. We could raise the hypothesis of a local adaptation and morphological differentiation without genetic structuring. We knew that different anatomic forms could partition their food resources by developing local adaptations (variations in feeding habits and spatial distribution) for the different habitats they used (Dynes et al. 1999). Indeed, Patton and Brylski (1987) have shown clearly body size variation in pocket gophers inhabiting alfalfa crops versus natural dry environments, Taylor et al. (2005) have underlined significant geographical differences in cranial shape between eastern and western Cape populations of Otomys saundersiae, occupying distinct ecological biomes. Several studies have shown the presence of local population-level differentiation in size and shape variation of molars, skulls and mandibles in mammals (Cardini et al. 2003, Cardini and O’Higgins 2004, Polly 2007) Moreover, the significant skull shape variation between populations showed that the morphological patterns evolved in Arvicanthis from Sudan are distinct enough to allow identification of populations (Rahman Abdel 2005). We also know already that African murids are able to vary their diet between seasons which suggests also a remarkable power of adaptation to local conditions in that group (Gliwicz 1987). In this study, some individuals would be more anthropophilic (using classical habitat) whereas others, pushed by the harsh environmental conditions of a drought (no breeding and intra-specific competition for food), were able to join a new habitat (swamp type) like a more humid area that served as a refuge and would have developed the first signs of a local adaptation. The 
differences of cranial shape could perhaps reflect the local adaptation without genetic structuring, ie morphological differentiation in the presence of gene flow, as a response to resolve problems of food availability (Herring 1993, Wood and Lieberman 2001, Debat and David 2001, Kingsolver et al. 2002, Piersma and Drent 2003, Lieberman et al. 2004). Because we had no analysis of stomach contents reported to verify the diet of each population, the explanation linking the shape differences to the fitness gain in the exploitation of resources available in the two environments is reasonable but speculative.

In summary, we propose that the widely-distributed Mastomys species exhibited a local morphological differentiation, probably due to different ecological situations. We speculate that the variability in the cranial characters (connected with the ability of feeding) could reveal preferentially a rapid and local adaptation based on the food availability. Since we suggested a potential differentiation process between populations, the two groups constituted even better models to understand the factors involved in the early stages of local adaptations. It would be very interesting to study these two populations in more detail to investigate the intra and inter-population morphological variability on a local scale and diet. The aim of such studies would be to provide a better understanding of this economically important species and its remarkable capability to proliferate.

Acknowledgements: We would like to thank Mr Ngouli, Mr White (COSTECH), Mr Severe (Wildlife Division Tanzania) and the field staff for their assistance in the "field" realization of this project (Research Permit No. 2003-152-CC-2003-83, Tanzania commission for Science and Technology, COSTECH). We are grateful to E. Lecompte, A. Delapré, M. Galan, C. Houssin, V. Aniskine, and Association 'Adrar des Iforas' (R. Cornette and S. Moulin) for the treatment and analysis of specimens. We thank F. Guy for his suggestions. We also acknowledge the reviewers for their attentive reading of the manuscript.

\section{References}

Airoldi J. P. and Flury B. K. 1988. An application of common principal component analysis to cranial morphometry of Microtus californicus and $M$. ochrogaster (Mammalia, Rodentia). Journal of Zoology, London 216: 21-36.
Alibert P., Fel-Clair F., Manolakou K., Britton-Davidian J. and Auffray J. C. 1997. Developmental stability, fitness, and trait size in laboratory hybrids between European subspecies of the house mouse. Evolution 51: 1284-1295.

Auffray J. C., Alibert P., Renaud S. and Bonhomme F. 1996. Fluctuating asymmetry in Mus musculus subspecific hybridization traditional and Procrustes comparative approach. [In: Advances in morphometrics. L. F. Marcus, M. Corti, A. Loy, G. J. P. Naylor and D. E. Slice, eds]. Ser. A Life Sci., Plenum Press, NY: 284: 275-283.

Baylac M. 2007. Rmorph: a $\mathrm{R}$ geometric and multivariate morphometrics library.baylac@mnhn.fr

Baylac M. and Friess M. 2005. Fourier descriptors, Procrustes superimposition, and data dimensionality: An exemple of cranial shape analysis in modern human populations. [In: Modern morphometrics in physical anthropology. D. Slice, ed]. Kluwer 145-166.

Beecher R. M., Corruccini R. S. and Freeman M. 1983. Cranio-facial correlates of dietary consistency in a nonhuman primate. Journal of Craniofacial Genetics and Developmental Biology 3: 193-202.

Belkhir K. 1999, GENETIX (v4.04): A Windows program for population genetic analysis.Laboratorie Genome, Populations: Interactions UPR $5000 \mathrm{du}$ CNRS, Universite Montpellier II, Montpellier (France).

Bookstein F. L. 1991. Morphometric tools for landmark data: geometry and biology. Cambridge University Press, NY: 1-455.

Bookstein F. L. 1996. Biometrics biomathematics and the morphometric synthesis. Bulletin of Mathematical Biology 58: 313-365.

Bouvier M. and Hylander W. 1996. The function of secondary osteonal bone. Mechanical or metabolic? Archives of Oral Biology 41: 941-950.

Cardini A. and O'Higgins P. 2004. Patterns of morphological evolution in Marmota (Rodentia, Sciuridae): geometric morphometrics of the cranium in the context of marmot phylogeny, ecology and conservation. Biological Journal of the Linnean Society 82: 385-407.

Cardini A., Tongiorgi P., Sala L. and O'Higgins P. 2003. Skull form and evolution in Marmota. [In: Adaptive strategies and diversity in marmots. R. Ramousse, D. Allainé and M. Le Berre, eds]. International Marmot Network, Lyon: 63-68.

Cook M. J. 1965. The anatomy of the laboratory mouse. .............., London and NY: $1-000$.

Cordeiro-Estrela P., Baylac M., Denys C. and Marinho-Filho J. 2006. Inter-specific morphological patterns of skull variation between two sympatric brazilian vesper mice: a geometric morphometrics assessment. Journal of Mammalogy

Debat V., Alibert P., David P., Paradis E. and Auffray J. C. 2000. Independence between developmental stability and canalization in the skull of the house mouse. Proceedings of the Royal Society of London 267: 423-430.

Debat V. and David P. 2001. Mapping phenotypes: canalization, plasticity and developmental stability. Trends in Ecology and Evolution 16: 555-561. 
Delany M. J. 1975. The rodents of Uganda. Trustees of British Museum (Natural History), London 746: 1-165.

Demby A. H., Inapogui A., Kargbo K., Koninga J., Kourouma K., Kanu J., Coulibaly M., Wagoner K. D., Ksiazek T. G., Peters C. J., Rollin P. E. and Bausch D. G. 2001. Lassa Fever in Guinea II. Distribution and prevalence of Lassa virus infection in small mammals. Vector Borne and Zoonotic Diseases 1: 283-297.

Denys C., Lecompte E., Lalis A., Cornette R., Moulin S., Makundi R., Machang'u R., Volobouev V. and Aniskine V. 2006. Small mammals systematics in Selous region, South Tanzania, Coast Province. Mammalia ..

Dobigny G., Baylac M. and Denys C. 2002. Geometric morphometrics, neural networks and diagnosis of sibling Taterillus species. Biological Journal of the Linnean Society $77: 319-327$.

Dos Reis S. F., Duarte L. C., Monteiro L. R. and Von Zuben F. J. 2002a. Geographic variation in cranial morphology in Trichomys apereoides (Rodentia: Echimyidae). I. Geometric descriptors and patterns of variation in shape. Journal of Mammalogy 83: 333-344.

Dos Reis S. F., Duarte L. C., Monteiro L. R. and Von Zuben F. J. 2002b. Geographic variation in cranial morphology in Trichomys apereoides (Rodentia: Echimyidae). II. Geographic units, morphological discontinuities, and sampling gaps. Journal of Mammalogy 83: 345-353.

Duarte L. C., Monteiro L. R., Von Zuben F. J. and Dos Reis S. F. 2000. Variation in mandible shape in Trichomys apereoides (Mammalia: Rodentia): geometric analysis of a complex morphological structure. Systematic Biology 49: $563-578$.

Duplantier J. M. 1988. Biologie Evolutive de Populations du Genre Mastomys au Sénégal. ..... thesis, Université des Sciences et techniques du Languedoc, Montpellier: $1-000$.

Dynes J., Magnan P., Bernathxez L. and Rodriguez M. A. 1999. Genetic and morphological variation between two forms of lacustrine brook charr. Journal of Fish Biology 54: 955-972.

Engström C., Kiliaridis S. and Thilander B. 1986. The relationship between masticatory muscle function and cranial morphology. II. A histological study in the growing rat fed a soft diet. European Journal of Orthodontics 8: 271-279.

Flury B. N. 1984. Common principal components in $\mathrm{k}$ groups. J.... Am.... Statist.... Assn 79: 892-898.

Galan M., Van Hooft W. F., Legrand D., Berthier K., Loiseau A., Granjon L. and Cosson J. F. 2004. A multiplex panel of microsatellite markers for widespread sub-Saharan rodents of the genus Mastomys. Molecular Ecology Notes 4: 321-323.

Gliwicz J. 1987. Niche segragation in rodent community of Africian dry savanna. Journal of Mammalogy 68: 169-172.

Gockel J., Harr B., Schlötterer C., Arnold W., Gerlach G. and Tautz D. 1997. Isolation and characterization of microsatellite loci from Apodemus flavicollis (Rodentia, Muridae) and Clethrionomys glareolus (Rodentia, Cricetidae). Molecular Ecology 6: 597-599.
Granjon L., Duplantier J. M., Catalan J. and Britton-Davidian J. 1997. Systematics of the genus Mastomys. Belgian Journal of Zoology 127: 7-18.

Gratz N. G. 1997. The burden of rodent-borne diseases in Africa south of the Sahara. Belgian Journal of Zoology 127: 71-84.

Herring S. W. 1993. Epigenetic and functional influences on skull growth. [In: The skull. Volume I. Development. J. Hanken and B. K. Hall, eds]. Univeristy of Chicago Press, Chicago: 153-206.

Hinten G., Maguiri T., Rossetto M. and Baverstock P. 1999. Isolation and characterization of microsatellite loci from the bush rat, Rattus fuscipes greyii. Molecular Ecology 8: 1351-1362.

Kiliaridis S., Engström C. and Thilander B. 1986. The relationship between masticatory muscle function and cranial morphology. I. A Cephalometric longitudinal analysis in the growing rat fed a soft diet. European Journal of Orthodontics 7: 273-283.

Kilonzo B. S., Mbise T. J. and Makundi R. H. 1992. Plague in Lusho district, Tanzania, 1980-1988. Transactions of the Royal Society of Tropical Medecine and Hygiene 86: 444-445.

Kingsolver J. G., Pfennig D. W. and Servedio M. R. 2002. Migration, local adaptation and the evolution of plasticity. Trends in Ecology and Evolution 17: 540-541.

Lalis A., Lecompte E., Cornette R., Moulin S., Machangu R. S., Makundi R., Aniskine V. M. and Denys C. 2006. Polymorphism of age population structure of two wild Mastomys natalensis (Rodentia: Muridae) Tanzanian habitat samples: a multicriteria comparison. Mammalia 70: 293-299.

Leary R. F. and Allendorf F. W. 1989. Fluctuating asymmetry as an indicator of stress in conservation biology. Trends in Ecology and Evolution 4: 214-217.

Leirs H. 1995. Population ecology of Mastomys natalensis (Smith, 1834). Implications for rodent control in Africa. $\mathrm{PhD}$ thesis, University of Antwerp, Brussels: 1-000.

Leirs H., Stuyck J., Verhagen R. and Verheyen W. 1990. Seasonal variation in growth of Mastomys natalensis (Rodentia: Muridae) in Morogoro, Tanzania. African Journal of Ecology 28: 298-306.

Le Louarn H. 1971. Détermination de l'age par la pesée des cristallins chez quelques espčces de rongeurs. Mammalia 35: 636-643.

Lieberman D. E., Krovitz G. E., Yates F. W., Devlin M. and Claire M. S. 2004. Effects of food processing on masticatory strain and craniofacial growth in a retrognathic face. Journal of Human Evolution 46: $655-677$.

Lima M., Stenseth N. C., Leirs H. and Jaksic F. M. 2003. Population dynamics of small mammals in semi-arid regions: a comparative study of demographic variability in two species. Proceedings of the Royal Society of London 270: 1997-2007.

Marchand H., Paillat G., Montuire S. and Butet A. 2003. Fluctuating asymmetry in bank vole populations (Rodentia, Arvicolinae) reflects stress caused by land- 
scape fragmentation in the Mont Saint Michel Bay. Biological Journal of the Linnean Society 80: 37-44.

Monath T. P., Newhouse V. F., Kemp G. E., Setzer H. W. and Cacciapuoti A. 1974. Lassa virus isolation from Mastomys natalensis rodents during an epidemic in Sierra Leone. Science 185: 263-265.

Monteiro L. R., Duarte L. C. and Dos Reis S. F. 2003. Environmental correlates of geographical variation in skull and mandible shape of the punare rat Trichomys apereoides. Journal of Zoology, London 261: 47-57.

Monteiro L. R., Lessal G. and Abe A. S. 1999. Ontogenetic variation in skull shape of Trichomys apereoides (Rodentia: Echimyidae). Journal of Mammalogy 80: 102-111.

Morris P. 1972. A review of mammalian age determination methods. Mammal Review 2 (3): 69-104.

Mossman A. C. and Waser P. M. 2001. Effects of habitat fragmentation on population genetic structure in the white-footed mouse (Peromyscus leucopus). Canadian Journal of Zoology 79: 285-295.

Mullin S. K., Taylor P. J. and Pillay N. 2004. Skull size and shape of Dasymys (Rodentia, Muridae) from sub-Saharan Africa. Mammalia 68: 185-220.

Mwanjabe P. S. and Leirs H. 1997. An early warning system for IPM-based rodent control in smallholder farming systems in Tanzania. Belgian Journal of Zoology 127 (Supplement): 49-58.

Mwanjabe P. S., Sirima F. B. and Lusingu J. 2002. Crop losses due to outbreaks of Mastomys natalensis (Smith, 1834) Muridae, Rodentia, in the Lindi Region of Tanzania. International Biodeterioration and Biodegradation 49: 133-137.

Nei M. 1987. Molecular evolutionary genetics. Columbia University Press, NY: 1-000.

Palmer A. R. and Strobeck. C. 1986. Fluctuating asymmetry: measurement, analysis, patterns. Annual review of Ecology and Systematics 17: 441-457.

Patton J. J. and Brylski P. V. 1987. Pocket gophers in alfalfa fields: causes and consequences of habitat-related body size variation. The American Naturalist 130: 493-506.

Piersma T. and Drent J. 2003. Phenotypic flexibility and the evolution of organismal design. Trends in Ecology and Evolution 18: 228-233.

Polly P. D. 2007. Phylogeographic differentiation in Sorex araneus: morphology in relation to geography and karyotype. Rus.... J... Theriology 6: 73-84.

Poulet A. R. 1980. Détermination de l'âge par la pesée des cristallins chez cinq espčces de rongeurs Muridés et Gerbillidés de l'Ouest de l'Afrique. Mammalia 44: 381-398.

Pucek Z. and Lowe V. P. W. 1975. Age criteria in small mammals. [In: Small mammals: their productivity and population dynamics. F. B. Golley, K. Petrusewicz and L. Ryszkowski, eds]. Cambridge University Press, Cambridge: $55-72$.

Rahman Abdel E. H. A. 2005. Systematics of Nile rats Arvicanthis (Rodentia: Muridae) from Sudan. PhD thesis, University of KwaZulu-Natal, South Africa: 1-000.

Raymond M. and Rousset F. 1995. Genepop (version1.2): population genetics software for exact tests and ecumenicism. Journal of Heredity 86: 248-249.

Rohlf F. J. 2000. On the use of shape scales to compare morphometric methods. Hystrix, Italian Journal of Mammalogy, New Series 11: 8-24.

Rohlf F. J and Slice D. 1990. Extensions of the Procrustes method for the optimal superimposition of landmarks. Systematic Zoology 39: 40-59.

Rousset F. 1997. Genetic differentiation and estimation of gene flow from $F$-statistics under isolation by distance. Genetics 145: 1219-1228.

Smithers R. N. H. and Wilson V. J. 1979. Checklist and Atlas of the Mammals of Zimbabwe Rhodesia. Museum Memoir $\mathrm{N}^{\circ}$ 9, National Museums and Monuments Rhodesia, Bulawayo.

Stenseth N. C., Leirs H., Mercelis S. and Mwanjabe P. 2001. Comparing strategies for controlling an African pest rodent: an empirically based theoretical study. Journal of Applied Ecology 38: 1020-1031.

Taylor P. J., Kumirai A. and Contrafatto G. 2005. Species with fuzzy borders: the taxonomic status and species limits of saunders'vlei rat, Otomys saundersiae Roberts, 1929 (Rodentia, Muridae, Otomyini). Mammalia 69: 297-322.

Watterson G. A. 1978. The homozygosity test of neutrality. Genetics 88: 405-417.

Weir B. S. and Cockerham C. C. 1984. Estimating F-statistics for the analysis of population structure. Evolution 38: $1358-1370$

Wood B. and Lieberman D. E. 2001. Craniodental variation in Paranthropus boisei: a developmental and functional perspective. American Journal of Physical Anthropology 116: $13-25$.

Yamada K. and Kimmel D. B. 1991. The effect of dietary consistency on bone mass and turnover in the rgowing rat mandible. Archives of Oral Biology 36: 129-138.

Yu H. T. and Peng Y. H. 2002. Population differentiation and gene flow revealed by microsatellite DNA markers in the house mouse (Mus musculus castaneus) in Taiwan. Zoological Science 19: 475-483.

Received 27 September 2007, accepted 18 February 2008.

Associate editor was P. David Polly. 